\title{
COLLIMATING DISCS AND BIPOLAR FLOWS IN SH 2-71
}

\author{
L. CUESTA and J. P. PHILLIPS \\ Instituto de Astrofísica de Canarias, E-38200 La Laguna, Tenerife, Spain
}

Sh 2-71 appears to represent a diffuse, ellipsoidal nebulosity extending over a range $\Delta \alpha \times \Delta \delta=1.7 \times 3 \operatorname{arcmin}^{2}$. Early spectroscopy by Glushkov et al (1975), and Chopinet and Lortet-Zuckerman (1976) suggested the presence of a high excitation central star, and Kaler (1983) has more recently determined Zanstra temperatures $\mathrm{T}_{z}$ (HeII) $>7.7 \cdot 10^{4} \mathrm{~K}$. The observed central star clearly constitutes a much cooler companion, and luminosity variations in this source have been attributed to binary eclipse.

We have recently undertaken a programme of high and low resolution spectrophotometry and NIR photometry of this source using the $2.5 \mathrm{~m}$ Isaac Newton Telescope (Observatorio del Roque de Las Muchachos), and 1.5 m Carlos Sanchez Telescope (Observatorio del Teide). As a consequence, we determine that:

(i) There is evidence for $\mathrm{H} \alpha$ velocities extending over a range $\Delta \mathrm{V} \sim 1026 \mathrm{~km}$ $\mathrm{sec}^{-1}$, with the principal emission peaks separated by $\Delta \mathrm{V} \sim 230 \mathrm{~km} \mathrm{sec}^{-1}$.

(ii) The kinematics of the exterior shell appear to be unusual; line velocities appear to shift rapidly over the nucleus, and mapping of the source reveals a distinct bilobal pattern, with high velocities to the $\mathrm{N}$ and $\mathrm{W}$, and lower velocities to the SE.

The kinematics of the exterior shell are interpreted in terms of a tilted disk outflow, in which material is ejected with initial velocity $\sim 19.6 \mathrm{~km} \mathrm{sec}^{-1}$, and subsequently decelerates towards the outer disk limits. Analysis of disk velocities and emission measures suggests overall mass-loss rates $\sim 10^{-6} \mathrm{M}_{\odot} \mathrm{yr}^{-1}$, although these values may have been higher in the past, whilst disk densities are likely to be low, and of order $\leq 10^{2} \mathrm{~cm}^{-3}$. The disk inclination is estimated to be $\geq 50^{\circ}$ to the line of sight.

It is proposed that this disk is also responsible for collimating the central high velocity wind into a bipolar flow, and we estimate associated core emission measures $1.6 \cdot 10^{6} \mathrm{~cm}^{-6} \mathrm{pc}$, together with densities $\mathrm{n}_{e}>2.1 \cdot 10^{4} \mathrm{~cm}^{-3}$. Mass loss rates appear to be $\mathrm{dM} / \mathrm{dt} \sim 2.0 \cdot 10-9 \mathrm{M}_{\odot} \mathrm{yr}^{-1}$.

Finally, it is suggested that the cooler companion may be filling its Roche lobe, and mass loss from this component is responsible for creating both the bipolar flow, and collimating disk. The earlier spectral type $\mathrm{B} 8 \mathrm{~V}$ attributed to this star is almost certainly wrong, unless derived distances to this source are grossly in error. We propose, rather, that nuclear fluxes be attributed to a blend of A7V and hot central star continua; a combination which enables reasonable fits to the optical continuum, and would result in binary eclipse amplitudes $\Delta \mathrm{m}_{p g} \simeq 0.75$ mags comparable to the luminosity variations found by Kohoutek (1979). 